\title{
Justicia Penal Juvenil
}

Jaime López Nuila

Abogado y Asesor Escuela de Derecho, UTEC

\section{Resumen}

Este artículo constituye una importante síntesis de la Justicia Penal Juvenil. Se ha tratado de destacar lo que deberá llamar la atención de la comunidad académica, para que sirva como fuente de consulta a los interesados y estudiosos.

\section{Concepto y desarrollo histórico de la infancia}

En lo que se llamaría un concepto de infancia, desde su origen antiguo -Grecia y Roma- en que se destaca la extrema dependencia al padre, quien ostenta poder de decisión sobre la vida y la muerte de su hijo y que conlleva también, la capacidad de disposición total sobre cualquier aspecto que se relacione con el futuro de aquel hijo, -su venta como esclavo-, llegaremos luego, a una situación en la que aquel concepto radical de Infancia es rescatado y humanizado por influencia de los principios cristianos.

Durante el tiempo comprendido entre los siglos XI y XV se conforma al fin un modelo de familia que buscaría garantizar la continuidad de la estirpe, limi- tando al fin algunos derechos del padre respecto al hijo, señalando límites respecto a edad del niño para responder penalmente de 10 a 14 años con una etapa de exclusión absoluta, y una pena atenuada para el menor de 14 a 16 años.

Es llegado el Siglo XVII en el que nace el concepto de Infancia como una categoría social, diferente al llamado adulto, unido a la idea de la "inocencia Infantil", en la que al niño se le ve por vez primera como la esperanza para un futuro mejor. Ya en el Siglo XVIII se cambian las razones de tener hijos. Ya no se tratará solo de perpetuar la estirpe con su carga de egocentrismo familiar, sino de dar y recibir amor al ser que decidimos dar vida. Es entonces cuando Rousseau marca en su Emilio la aparición temprana del concepto de la "dignidad del niño" con sus consecuencias finca- das en una especial y cada vez mayor atención al Infante.

En el Siglo XIX, pleno de desarrollo en nuestras concepciones humanitarias, encontraremos la idea de que el Estado está en la obligación de proteger al niño. Se comienza a rechazar el castigo corporal del menor, se amplían y masifican los medios de enseñar al niño imponiendo la escolaridad obligatoria, y se origina el concepto de institución que cuidará del menor necesitado. En esta etapa, se crean los primeros Tribunales Tutelares de Menores, lo mismo que sociedades que tratan de abrigar y proteger al niño y se enviste al Juzgador con un papel que trasciende de Juez, para convertirlo casi en padre de familia sustituto para el menor que comete un delito. La salud mental y física del niño es tema de primer interés para el Estado. Se llega al Siglo XX con 


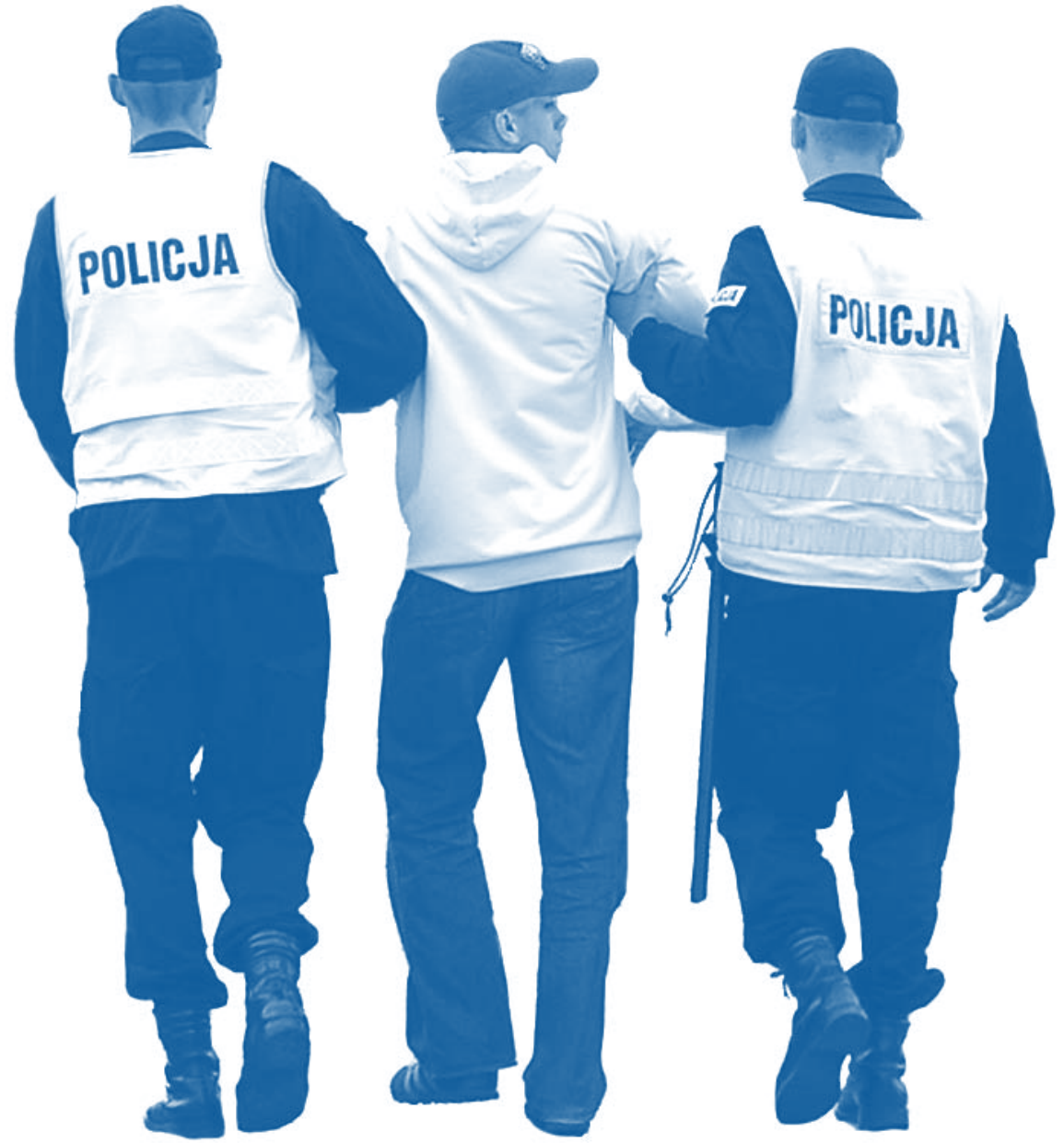

su plenitud de decisiones a favor de la niñez, desde los esfuerzos para ampliar y mejorar una ciencia médica especial para preservar la vida del niño -la Pediatría- y la reducción de índices de mortalidad infantil. Además, se legisla abundantemente en leyes para la infancia, de carácter nacional e internacional. En 1924 la llamada Sociedad de las Naciones aprueba la Declaración de Ginebra, primer documento que contiene disposiciones del Derecho Internacional, que se refieren al niño. Así se llega a finales del Siglo XX a la firma de
La lejana figura paternalista del Juez que debía proteger al menor se convierte, merced al reconocimiento de los derechos del niño que impulsa la Convención, en una institución representada por autoridades especiales para menores, que rigen su procedimiento sobre base a normas diferentes y singulares, que obedecen ciegamente al principio conocido del Interés Superior del Menor. Esta es la esencia del Derecho Penal Juvenil.

Respecto al procedimiento en el Juicio Penal a menores, hay que señalar que de la Doctrina de la situación Irregular (menor en conflicto con la ley penal o menor infractor) y que alude a la falta cometida por el niño, se llega a la Doctrina de la Protección Integral, entendida como un estadio superior de desarrollo del esfuerzo por garantizar mejor al joven que transgredía la norma, tratando que conserve en el mayor grado sus mejores derechos. Se ha llegado actualmente a concebir una Protección Universal de los Derechos del Niño, que adquieren vigencia, y son respetados, en el lugar y sitio en que se encuentre, sin distingos de nacionalidades y territorios, porque el fin es conseguir la aceptación universal de los Derechos del niño.

Mauricio González Oviedo, Sociólogo de la Universidad Nacional de Costa Rica y Consultor Internacional de la UNICEF 
para El Salvador y otros países ha desarrollado la presente temática, y respecto al concepto de la infancia, aclara que la llamada doctrina de la protección integral del menor no existe, sino lo que él Ilama Doctrina de las Garantías, que se basa en la llamada ciudadanía social que es fundamentada en todas las "teorías garantistas" conocidas. González Oviedo, pretende llegar al desarrollo máximo de la dogmática jurídica referida a las garantías que deben rodear al menor infante 0 adolescente cuando viola la ley.

Así, Oviedo razona que para arribar al concepto de Ciudadanía de la Infancia y la adolescencia se deben interpretar primero los conceptos de Ciudadanía Social, Civil y Política. Para él la llamada Ciudadanía de la Infancia y la Adolescencia es una ciudadanía ampliada, resumen y conjunto de Derechos Sociales, Civiles y Políticos, más los Especiales Derechos del niño y del adolescente, que contiene incluso, todos los cuidados que se le deben al sujeto que está en el vientre de la madre, controles prenatales, vacunas, incluido su Registro Civil, para acreditar su vida como sujeto pleno de garantías en su relación con la Sociedad que lo recibe y el mundo al que ha llegado.

Oviedo explica que llegado ese niño al mundo, comienza un mosaico de relaciones sociales, con sus padres y fami- lia, entorno social, escuela y sociedad en que se desarrolla. El ser humano, y por supuesto ese niño, actuará en el futuro conforme a los valores que recibe en la infancia y en el proceso de formación de sus años primeros. Este proceso de formación actuará conforme las relaciones que conoce, de rechazo o anulación, de comprensión y amor. No deberemos expulsar, por ejemplo, a un niño de una escuela por tener un comportamiento diferente al resto o por las fallas y errores de los adultos encargados de formarlo.

Ese menor y niño expulsado, segregado y rechazado, será luego excluido por el Estado que no le abrirá espacios para su participación plena en la comunidad. Ese niño podría ser mañana el pandillero que encuentra su familia en el grupo que integra la mara o la pandilla, también podría ser el temido delincuente que destroza vidas a las que nunca aprendió a valorar y respetar.

\section{Instrumentos jurídicos}

La Convención de 1990 es la suma histórica de Derechos del niño recogidos en variados instrumentos jurídicos. Se puede decir, que formalmente, la Convención sobre los Derechos del niño empieza a estructurarse en 1924 cuando la llamada Liga de las Naciones produce el primer Documento que, en forma relevante y con carácter Internacional, reconoce Derechos Inherentes al niño. Después, en 1959 las Naciones Unidas adoptan la Declaración de los Derechos del Niño siempre con la forma de instrumento para ser aplicado Internacionalmente, pero que tiene una naturaleza más específica en cuanto al personaje a quien protege que aquél pronunciamiento que le precedió.

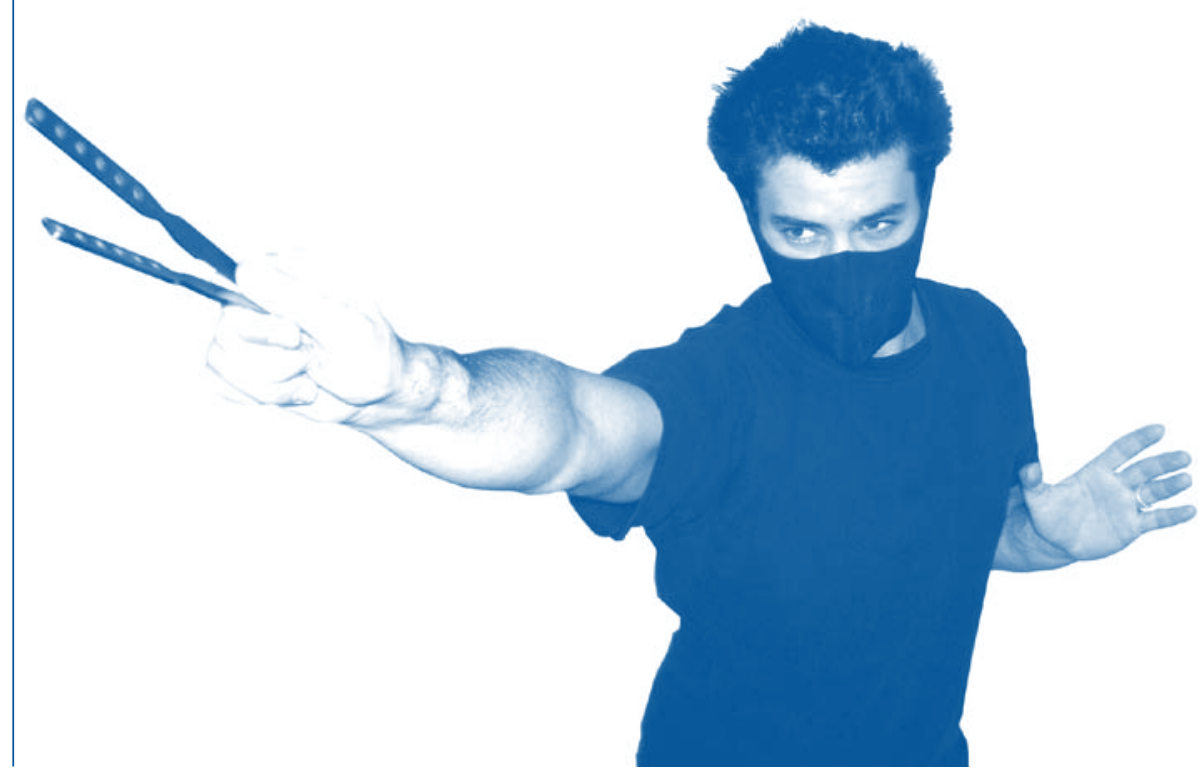


Luego del año 1959 se produce la aprobación de Resoluciones y Acuerdos singulares siempre en provecho de la niñez que, inscritos en el marco general de los Derechos Humanos se proponen viabilizar el disfrute de determinados derechos y garantías por parte del niño. Finalmente se llegará a la declaratoria y aprobación de la llamada Convención sobre los Derechos del Niño, que excepcionalmente, gozaría de una aprobación unánime y sin demoras, no siempre presentes características en las organizaciones Internacionales. Esa arrolladora bienvenida en la Comunidad Internacional al Documento que contiene los Derechos y garantías del Infante, importa no obstante, según la Mary Beloff, a la que seguimos en estas consideraciones, verdades inconfesas y a menudo negadas, en el ambiente de la Comunidad Internacional de Naciones.

Según Beloff, la Convención sobre Derechos del Niño no es un mecanismo fuerte de control, de cara al comportamiento particular de cada Estado, responsables principales para el cumplimiento de aquellos derechos; carece asimismo de verdaderos y eficaces instrumentos de exigibilidad para el cumplimiento de Derechos Económicos, Sociales y Culturales a favor del menor. Además, la Convención contiene disposiciones que autorizan conductas y acciones que anulan ó limitan derechos del niño, como aquellas que regulan el trabajo Infantil, y, finalmente, la misma Convención exhibe una criticable ambigüedad en su texto cuando señala los derechos y la forma de su ejercicio, de forma que finalmente, termina por autorizar la negación ó la reducción de los tales derechos y garantías del niño.

La crítica mas acentuada a las deficiencias en el texto de la Convención están señaladas al afirmar que "solo es un enunciado político y de propaganda" incapaz de producir por sí sola, profundas transformaciones sociales en el marco de garantías para la vida del niño, ya que mas allá de su particular estilo declarativo, solo implica una forma de superar viejas prácticas custodiales y represivas encubiertas en el modelo tutelar, no significando por lo mismo, la vigencia y respeto de la aplicación práctica de los Derechos del niño que la misma Declaración expresa.

Pero de todos modos, lo cierto es que la Declaración contenida en la Convención sobre los Derechos del Niño, no tiene la naturaleza de una simple declaración de principios porque su contenido representa el nivel mínimo de garantías y Derechos que protegen la Infancia, y que, teniendo también carácter normativo incluso de superior jerarquía que la Ley secundaria, debería llevar consigo la obligación de hacer efectiva su prestación y obediencia en su cumplimiento.

Beloff reconoce que la Convención implica, por lo menos, un cambio de paradigma en la manera de conocer al niño, y que su impacto en la comunidad in-

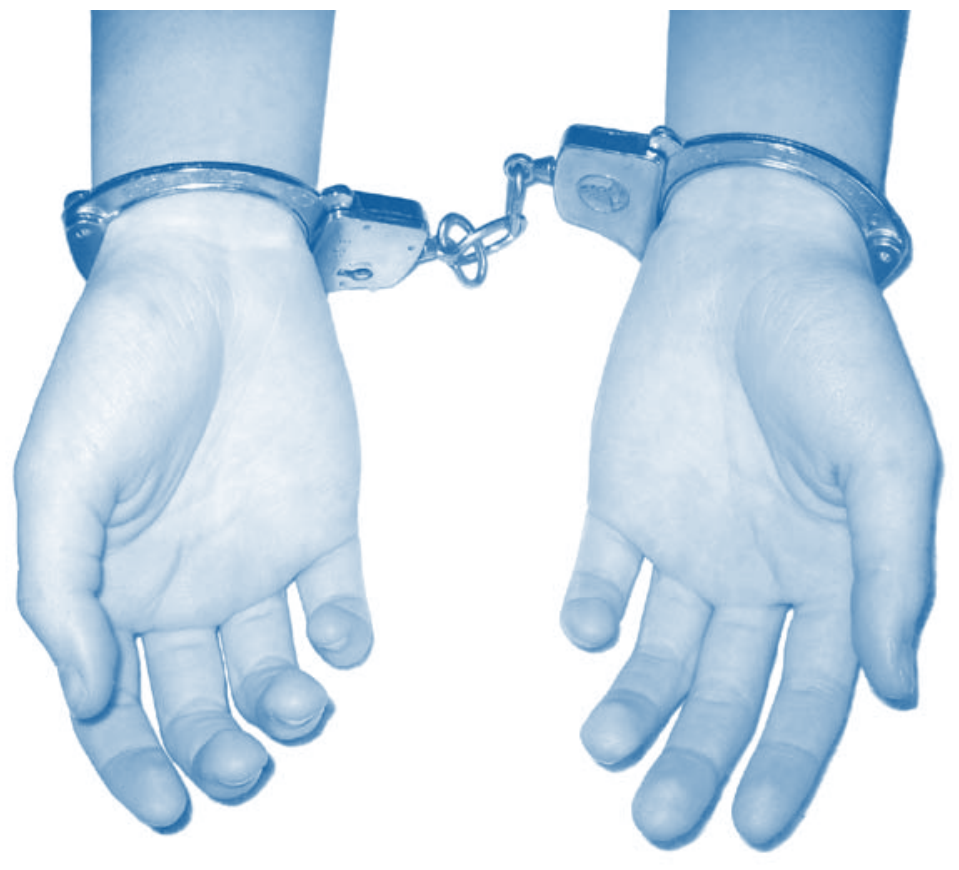


ternacional ha traído el cambio de algunas prácticas socio-jurídicas que eran comunes antes de su vigencia. Pero de igual forma reconoce que no se puede considerar al Tratado que da vida a la convención como un Tratado de Ruptura, en el sentido de suprimir todas las practicas previas que desconocen aquellos Derechos del menor, primero, porque no tiene la naturaleza de un Tratado duro ó inflexible, tanto `por el número mínimo de países que le dan vida con el carácter de una Convención de amigos, como también y sobre todo, porque no tiene un Capítulo de definiciones y tampoco cuenta con mecanismos duros de exigibilidad de los derechos que le reconoce al niño.

La profesora Especialista en Harvard y Consultora Internacional sobre temas referidos a la infancia y al Derecho Penal Juvenil aclara, que las debilidades del Convenio sobre Derechos del niño ya señalados, obliga a consultar otros cuerpos normativos para superar las deficiencias del Sistema contenido en la Convención. En el ámbito de América se remite a la Convención Americana sobre Derechos y Deberes del hombre; la Convención Americana sobre Derechos Humanos; el Protocolo de San Salvador, la Declaración de los Derechos del Niño y otros. El marco jurídico de los Derechos de la Infancia se completa con diversos instrumentos que ayudan a interpretar el Convenio de protección al Niño, supliendo a veces las faltas de definición que dijimos antes, afecta a la Convención. Así, encontramos a las llamadas Reglas de Tokio que son las Reglas mínimas para la aplicación de medidas diferentes a las privativas de libertad; las directrices de Naciones Unidas para la prevención de la delincuencia juvenil conocida por Directrices de Riadh; las Reglas de N.U. para la protección de menores privados de libertad; y además las Reglas mínimas de N.U. para la Administración de la justicia de menores.

En este punto de la vigencia, reconocimiento y aplicación práctica del Sistema que provee la Convención de los Derechos del niño se destaca la participación en litigios recientes ante la Corte Interamericana de Derechos Humanos ó Pacto de San José, que dispone de mecanismos de Control Estatal fuertes que posibilitan un reclamo mas efectivo de los Derechos del niño cuando son vulnerados, fijando al final con sus resoluciones, estándares nuevos por intermedio de la jurisprudencia, con la subsiguiente exigencia de cumplimiento estricto de los fallos pronunciados contra los Estados, demandados por violaciones a derechos de la niñez. Esta integración del Derecho protector del menor ya ha cobrado vigencia en la Jurisprudencia de la Corte Interamericana de los Derechos Humanos ante la que en el año 1997 se produce Sentencia que condena al Estado de Guatemala, y que la Beloff reseña en su ponencia “Los Derechos de los niños en el Sistema Interamericano de protección de Derechos Humanos", y que representa un ejemplar precedente de condena al Estado, demandado por ejecuciones sumarias de personas, entre ellas, algunos menores de edad. Lo bueno del fallo, es la forma en que se logra integrar la protección de la Convención sobre los Derechos del niño, con el Art. 19 de la Convención Interamericana de Derechos humanos, produciendo una Resolución que señala un nuevo estándar de protección a los Derechos de la Infancia, como cuando califica al Derecho a la vida como un derecho de prestación positiva por parte del Estado, lo que significa que parte del compromiso del Estado es que está obligado a garantizar al niño una vida digna de ser vivida.

La litigación práctica en el fuero de la Corte Interamericana ha llegado hasta ahora, al reconocimiento de la violación por el Estado Argentino en el caso de una detención ilegal con consecuencias extremas, que viola el Derecho a la libertad personal, a las garantías judiciales, al derecho de recibir ayuda por parte del Estado, al debido proceso con respeto a garantías judiciales, a la integridad personal, y en su caso, al Derecho a la vida. La Corte resuelve con la condena al Estado infractor a repara- 
ciones pecuniarias, al reconocimiento público de su propia responsabilidad, como ente obligado a tutelar y proteger Derechos, y además a continuar hasta su conclusión el debido proceso para establecer responsabilidad en casos concretos.

Respecto de la aplicación integrada de la norma jurídica del Convenio sobre Derechos del niño, es interesante mencionar el caso litigado en Sede de la Corte Interamericana, de menores contra el Estado Dominicano, por irrespeto a sus Derechos humanos a la nacionalidad, a la Igualdad ante la ley, al Derecho a la personalidad y Derecho al nombre por la negativa del Registro correspondiente, a asentar el nacimiento de menores que, nacidas en República Dominicana, y de madres dominicanas, eran no obstante de ascendencia Haitiana, en lo que se percibe como Acto de discriminación racial.

Otro caso paradigmático de Jurisprudencia de la Corte Interamericana de Derechos Humanos que establece nuevos estándares Internacionales de respeto de Derechos Humanos y la exigibilidad de la prestación positiva de Derechos de carácter Económico y Social, con lo que se busca dar contenido a la protección integral de Derechos de la niñez que no se encuentra regulado en la Convención, es el caso del Instituto de Reeducación del menor de Paraguay contra el Estado Paraguayo, que reseña la violación de derechos, entre otros, de la vida de Internos, niños en conflicto con la ley, que no llena requisitos en la infraestructura del establecimiento

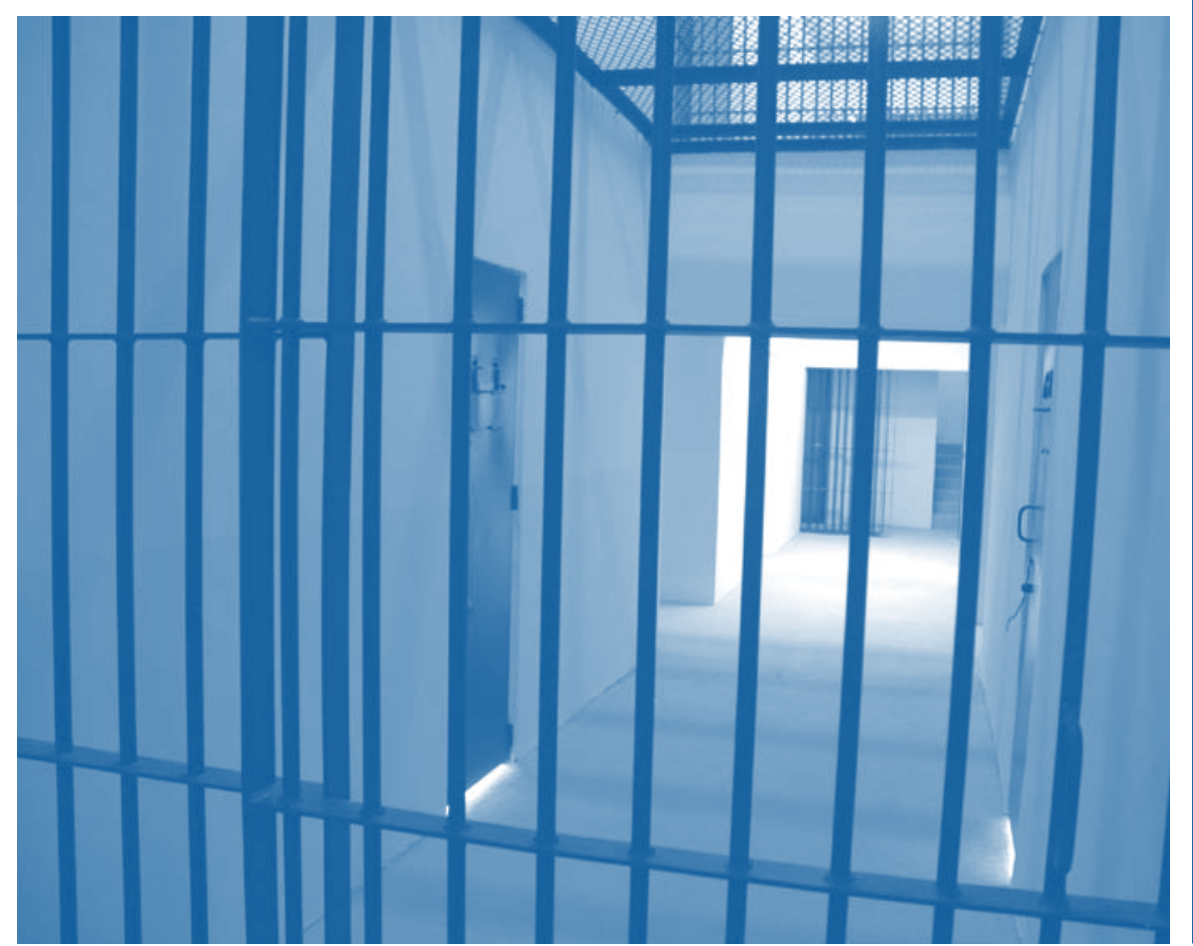

carcelario. Entre trámites judiciales de un Recurso de Exhibición personal de carácter general, sin que se modifiquen las precarias condiciones denunciadas, se produce un siniestro que produce la muerte de varios internos menores. La condena al Estado Infractor reconoce que la obligación para con el niño y su exigibilidad llega para el Estado, mas allá de su participación directa en acciones que originan la violación del Derecho a la vida, ya que esa "obligación positiva" en este caso, forma parte de la obligación de proveer las condiciones requeridas para evitar que el suceso cause la pérdida de vidas como en el caso denunciado.

Para concluir esta consideración puramente Institucional del marco legal que defiende los Derechos del niño se debe expresar, que además de la Integración del Derecho relacionado con ese problema, el posicionamiento de esta normativa tan particular tiene que contar con el auxilio de resoluciones al nivel de instrumentos y Cortes de Justicia Regionales, y al mismo tiempo, con especial compromiso y paciencia, obtener el acatamiento estricto de la norma jurídica que protege a la infancia por parte de los Estados compromisarios, hasta llegar al punto en que tal respeto de las garantías y Derechos del niño sea la norma y el estándar general, y no la excepción, como lo es en el momento presente. 